\title{
EN TORNO A LA ENSEÑANZA DE LA ECONOMIA*
}

\author{
Jaime Jaramillo Uribe
}

\section{Exigencias de la época}

Hace todavía cuatro lustros el país no sentía la necesidad ni la urgencia de tener una clase dirigente especializada en el análisis de los hechos económicos y en el manejo de su economía. Por consiguiente, tampoco la Universidad llegó nunca a contemplar la posibilidad de una enseñanza académica y sistemática de la técnica y la ciencia económicas ni a plantearse el problema de la formación de profesionales en este campo de la cultura.

Para esa etapa de nuestro desarrollo, caracterizada por una economía simple, en la que prácticamente no existía aún un mercado nacional unificado, ni una industria fabril, ni un sistema monetario complejo, el hombre de acción, el "práctico", el empresario hechura de sí mismo o el abogado que aprendió en nuestra Universidad colonial algunas nociones de economía política novecentista tomadas de los libros de Mill, Leroy Baulieu o Charles Gide eran todavía los gestores indicados y más o menos eficientes de la actividad económica.

Pero a partir de 1930 la economía colombiana da un salto no simplemente cuantitativo sino cualitativo y por esta circunstancia se ha sentido la necesidad de acudir al técnico y al profesional de la economía tanto en las esferas privadas como en las del Estado. Desde entonces han comenzado a surgir entre nosotros escuelas e institutos especializados que se han impuesto la tarea de formar los nuevos cuadros profesionales. Se ha visto que la nueva realidad económica no puede ya, no digamos dominarse, sino conocerse en sus resortes esenciales sin la contribución del especialista y que éste no puede formarse sino en instituciones dedicadas exclusivamente a la enseñanza de la economía, servidas por un profesorado adecuado.

Es verdad que la economía es todavía una ciencia imperfecta, cargada de problemas, llena de oscuros arcanos a cuyo fondo aún sus más conspicuos cultivadores dudan de poder llegar triunfadores. Pero la existencia de problemas insolutos y de grandes enigmas no quiere decir inexistencia de una ciencia, ni falta de rigor ni menos aún ausencia de utilidad. En realidad toda ciencia es más bien un conjunto de problemas que una colección acabada de soluciones. Mucho más en una disciplina enderezada a la comprensión, el análisis y la previsión del desarrollo económico, fenómeno complejo en que se cruzan factores imprevisibles de la acción humana y factores de tipo natural gobernados por verdaderas leyes, sujetos a causalidad rigurosa y en alta medida calculables en forma matemática. Pero no obstante sus imperfecciones, la sociedad no puede pasarse sin los economistas ni la universidad ha podido evadir su presencia. Sin ser una ciencia acabada - en rigor ninguna ciencia llega a serlo la economía ha realizado en los últimos cuarenta años progresos inmensos. Sobre todo a partir de la primera guerra mundial sus fundamentos se han transformado y sus métodos, sus análisis y sus conceptos se han afinado en tal forma, gracias a nuevos hechos y a los renovados avances de la teoría, que ya es necesaria una

\footnotetext{
* El Financiero, revista colombiana de economía y finanzas, año 1, No. 6, diciembre de 1951, pp. 41-44. 
vida para dominarlos y hasta podríamos decir insuficiente. Los viejos textos tienen apenas un valor histórico y los antiguos principios simples, los ingenuos expedientes de la oferta y la demanda y las descarnadas informaciones técnicas no capacitan a nadie para orientarse con alguna seguridad en tan complejo universo.

Los análisis matemáticos en primer lugar, son indispensables. Hoy no se concibe un técnico ni un economista que no posean una sólida preparación matemática y que no dispongan de un extenso cuerpo de conocimientos auxiliares en materia geográfica, estadística, financiera e incluso histórica. Pensar correctamente ese intrincado mundo de las relaciones económicas ha hecho indispensable el adiestramiento especializado de la mente, adiestramiento que no puede adquirirse ya en la práctica de los negocios ni absorberse como conocimiento marginal de otras profesiones. Las escuelas de economía no han surgido como un capricho de espíritus académicos, sino como una exigencia del medio social, exigencia que ha llegado a nuestros propios terrenos. Así como la escuela pública, el colegio y la universidad que enseña profesiones fueron apareciendo históricamente cuando el volumen de conocimientos científicos y técnicos no podía adquirirse en la experiencia cotidiana ni en contacto con la familia, así la escuela de economía ha surgido en momentos en que el mundo de lo económico se ha hecho inabarcable por obra de la pura "praxis" y cuando la ciencia y la técnica económicas han alcanzado un desarrollo sin precedentes.

Sin embargo, hemos de confesar que si bien es cierto que el país se ha dado cuenta de esta urgencia de la época y para satisfacerla ha ido creando centros docentes oficiales y privados dedicados a la enseñanza económica, no es menos evidente que hasta el presente hemos atravesado por un período de tanteos, confusiones y desaciertos. El fenómeno es por lo demás explicable, pero la solución no consiste en ocultarlo o en perseverar en orientaciones erradas por prejuicios o vanidades. En nuestra opinión, tratarlo con sinceridad es la mejor manera de servir al país y a las nuevas generaciones que ven en la economía un nuevo e ilimitado campo de estudio y acción.

Es natural que en la primera etapa se hayan presentado en el país confusiones y deficiencias. Hemos poseído una mediana tradición académica en ciencias naturales, en ciencias físico-matemáticas aplicadas, en medicina y derecho, pero carecemos de todo antecedente en ciencias sociales en el sentido moderno y en primer término en economía. Por eso el más grande escollo que han tenido las escuelas superiores de esta disciplina ha sido el de la falta de un profesorado especializado y de una dirección que entienda en sentido estricto cuáles son las necesidades en este campo y cuáles orientaciones científicas internas que deben darse a estos estudios. Por esta circunstancia nos ha parecido conveniente intentar un balance muy general de lo existente y someter a críticas algunas ideas que flotan en nuestro ambiente, con el ánimo único de contribuir a un nuevo examen del problema en el sentido universitario y docente.

\section{Expertos técnicos y economistas}

Lo primero que deben tener claro nuestros centros de enseñanza económica es qué tipo de profesional se proponen formar. O en otros términos, cuál es su verdadero objetivo. Y a este respecto nos parece que debe establecerse una diferencia entre el "experto técnico" y el "economista". Un economista es ante todo un hombre teórico. Teórico, no el sentido vulgar en que solemos entender este término, cargándolo de cierto matiz peyorativo o asimilándolo a falta de sentido de la realidad. La teoría es lo más real que existe y el verdadero hombre 
teórico —que lo es todo gran científico- el ser que menos utopías forja y que menos ilusiones se hace. Aquí hablamos del "economista" como un teórico en el sentido de que sus problemas son estrictamente científicos, es decir, que se mueve en el plano de los problemas generales, de las relaciones de los diversos fenómenos considerados como un todo, de los principios de la ciencia económica.

Un "experto técnico", en cambio, es alguien que maneja con destreza ciertos métodos de trabajo, de general aplicación, eficaces para la solución de problemas invariables y en alta medida de sentido mecánico. Toda técnica es en general mecanismo. La diferencia entre "experto técnico" y "científico" se da en todas las ramas de la actividad cultural. En matemáticas, en biología, en física, en medicina, en derecho y en economía. En todas estas disciplinas hay el práctico y el sistemático, el conocedor de los instrumentos mecánicos de control y el investigador, descubridor, analista de hechos conexos, creador de leyes y de principios generales. Ambos tipos son indispensables para la sociedad, pero ésta los necesita y los puede producir en proporciones diversas y siguiendo métodos diferentes. Es fácil y muy necesario para un país tener muchos técnicos; pero es difícil aunque igualmente necesario tener muchos economistas.

Ahora bien, partiendo de esta clasificación entre "experto" y "economista" es que a nuestro juicio deberían organizarse las escuelas de economía y sus respectivos planes de estudio. Algo más, creemos que en el estado actual de las necesidades nacionales y de los recursos materiales y docentes del país, los centros de enseñanza económica deberían ser institutos dedicados a la formación de "expertos técnicos", abandonando la pretensión muy loable pero utópica y excesiva de hacer de cada estudiante un "economista". No sostenemos con esto que el país no necesita ni pueda formar ,economistas. Ni que un experto técnico no deba tener el dominio y la información mínima de ciertos aspectos teóricos y generales de la economía, en la misma forma en que el economista necesita técnica. Pero dentro de las condiciones reales de nuestro medio habrá que utilizar los escasos recursos — simple cuestión también de economía- en la formación de "expertos" y dejar que los "economistas" se formen como autodidactas o salgan a especializarse en el exterior.

Lo que el país necesita con mayor urgencia en esta etapa de su desarrollo, es un grupo capaz y numeroso de técnicos. Expertos en administración industrial, en control racional de empresas, contadores, gerentes, hombres que dominen con precisión la técnica de los negocios, la organización monetaria y bancaria, que conozcan el mecanismo del comercio nacional e internacional, que sepan analizar la situación financiera de una empresa y enfrentarse a sus problemas de organización. Todo esto es técnica esencialmente y pertenece al dominio del "experto" más que al campo del "economista" que es un teórico y un hombre de ciencia.

Sobre la base de este objetivo concreto, deberían reajustarse las orientaciones y planes de estudio de nuestras escuelas de economía, podando sus ambiciones de encomiables pero utópicos planes de cultura universal ${ }^{48}$. Mucha estadística, muchas matemáticas comerciales, mucha contabilidad de costos, idiomas modernos, administración de empresas, geografía económica y las bases esenciales de la economía teórica. Pero ante todo, técnica, técnica

\footnotetext{
${ }^{48}$ La Facultad de Administración Industrial del Gimnasio Moderno de Bogotá parece que se orienta con bastante éxito por la formación de lo que aquí hemos denominado "experto técnico". Desconocemos los planes y tendencias de la recientemente fundada escuela de economía de la Universidad de los Andes.
} 
concentrada que frustre el "dilatentismo" y la dispersión utopista. Las materias histéricas y políticas deben reducirse al mínimo e incluso suprimirse. Comprendemos que un economista deba conocer la historia económica universal y la historia minuciosa del pensamiento económico. Entendemos que debe conocer la historia del capitalismo industrial y de la época mercantilista, y si conoce la economía entre los aztecas y los incas tanto mejor. No hay economista sin gran cultura y la historia está llena de ejemplos desde Cantillon hasta Marshall y John Maynard Keynes. También debe el economista dominar los campos de la sociología, como disciplina científica se entiende y no como concepción del mundo o como un vago equipo de bien intencionados principios de redención social, lo mismo que el derecho y ante todo la matemática y la lógica.

Pero es materialmente imposible, y además innecesario, pretender que estos conocimientos son indispensables para un experto técnico y que ellos puedan ser absorbidos por un principiante, en el curso de pocos años y partiendo de una preparación tan precaria como la que suministra nuestra enseñanza secundaria. Algunas de nuestras escuelas de economía tienen cátedra de Planificación, Política Social, Economía en la Prehistoria, Política Económica, Historia de las doctrinas políticas y económicas, etc., etc. El resultado de un plan de estudios y de una orientación así concebidos, es una profunda dispersión y una deficiencia de la preparación práctica, por razones obvias. En primer lugar, porque no existen en el país profesores especializados y dedicados a la enseñanza de estas cátedras. En segundo lugar, porque ese es el camino más propicio para la vaguedad y para mezclar la auténtica disciplina económica con otros conocimientos que pueden ser auxiliares de la economía pero que ni la sustituyen ni son la economía.

\section{Errores y prejuicios}

Hay todavía un problema que debemos mencionar en estas notas sobre la enseñanza de la economía en el país. Es algo que atañe a la enseñanza de muchas otras disciplinas científicas, pero que en la economía es excepcionalmente agudo y lleno de graves consecuencias por la inmadurez misma de la ciencia económica y por la debilidad de nuestra formación cultural. Nos referimos al nebuloso relativismo que domina en quienes suelen ocuparse de la enseñanza económica y a las numerosas confusiones de su esencia con la de otras disciplinas que se le parecen o le sirven de auxiliares. La reducción de la economía a una mezcla informe de otras ciencias, o a un conjunto de puntos de vista ideológicos (éticos, políticos, etc.) o a un cuerpo de preceptos que se dicen ser válidos únicamente para un país dado, para un medio o una situación histérica determinada, no sólo se respira en los centros llamados cultos, lo que no sería muy grave ni es privilegio nuestro, sino que domina en los centros universitarios debido a la falta de una tradición académica en estas materias y la precaria formación científica general de la mayor parte de quienes sólo ocasionalmente se ocupan de labores docentes. Y mientras perduren estas confusiones y estos puntos de vista, no habrá auténtica enseñanza económica en Colombia. Por eso nuestro deber es poner de presente los problemas que estas orientaciones implican, aunque ello sea una tarea ingrata y aunque la solución del mal no esté a la mano ni sea fácil.

La primera fuente de error está en el concepto de economía que predomina entre nosotros, heredado de una situación superada hace años en Europa y los Estados Unidos. Nuestros profesores y economistas siguen definiendo la economía a la manera del siglo pasado, como la ciencia que se ocupa de la riqueza, o como la teoría de la producción, la distribución y el consumo de ésta, o como la ciencia de la abundancia de bienes para el 
mayor número, etc., etc. Falla desde un principio la misma definición de la disciplina en éste que por ser país de gramáticos y juristas debería ser amigo de las distinciones, clasificaciones y definiciones rigurosas, poseedor en alto grado del sentido de la precisión, del "esprit de géometrie" de que hablaba Pascal.

De una falsa definición del campo de problemas propios de la economía, nacen casi todos los extravíos de nuestra enseñanza económica. Es ese el origen del grave mal de que se confunda la economía con disciplinas que pueden auxiliarla pero que no son la economía. Por ejemplo, que a veces se la confunda con la historia económica, o con la historia de las doctrinas, confundiendo la teoría general de una ciencia con la historia de su desenvolvimiento. Pero el mayor peligro y la mayor fuente de errores es la conversión de la economía en una ideología política o ética, o la afirmación, tan cara al hombre común, al lego y al "diletante" de que hay una ciencia económica para cada país, para cada continente y para cada circunstancia histórica. La afirmación tan corriente de que necesitamos una teoría económica —nos referimos a una teoría o ciencia económica, no a una organización económica, cosa diferente- propia para los pueblos latinoamericanos atrasados y semicoloniales, etc., tiene su origen en el desconocimiento de lo que es la ciencia económica y es fuente de vaguedad y "diletantismo".

De este mismo error inicial, de este relativismo tan caro a los espíritus poco rigurosos, surge la creencia tan corriente y fatal para la preparación científica de que existe una teoría económica cristiana, o una socialista en oposición a una capitalista, pongamos por caso. Quienes así piensan, confunden el análisis puro de los hechos económicos con el examen o el anhelo de que las formas de vida social o política, comprendiendo las formas de apropiación de la riqueza y aspectos como las relaciones de trabajo se organicen en determinadas formas. Pero la economía es un conjunto de normas puras y lógicas, en cierta manera de formas vacías como el análisis matemático, que se ocupan de cosas y relaciones entre cosas y apetencias personales. La afirmación de que existe una ciencia económica soviética, capitalista, europea o latinoamericana, es tan válida como la que se refiere a la existencia de una física capitalista y una socialista. Todo eso constituye una verdadera mitología, un cuerpo de tabús que no han hecho sino sembrar confusión en la mente de las gentes que asisten a las aulas sin prejuicios, ansiosas de adquirir una preparación profesional.

No es este el momento ni tenemos aquí el espacio para plantear el problema fundamental del campo propio y exclusivo del análisis económico, pero no está de sobra recordar que la única definición lógica de la disciplina económica es la que la demarca como la ciencia que estudia la relación entre fines o necesidades y medios escasos — Robbíns, Cassel_y que el núcleo central y básico de esta ciencia es el de escasez. Lo que de ahí sigue no es sino un cálculo de relaciones basadas en que hay un conjunto de necesidades y unos medios limitados para satisfacerlas. La escasez es la realidad que engendra toda conducta y toda teoría económicas. No hay problema económico con bienes que son superabundantes, que no deben ahorrarse en alguna manera y que no nos presentan usos alternativos. $Y$ este punto de partida es el mismo y tiene la misma validez en la Inglaterra laborista, en los Estados Unidos capitalista, en la Rusia soviética y en los países semicoloniales de Asia o América Latina. Esto, desde luego, no quiere decir que los datos únicos de una realidad económica y social particular no entren con su ponderación específica en el análisis económico. Pero entran simplemente como un dato en un examen formal cuyo concepto central es la escasez. El mundo de lo económico es altamente complejo y en medida muy considerable imprevisible 
e inaprehensible por fórmulas exactas. Los llamados datos concretos de una realidad histórica, de un momento dado o de un país en particular, los hábitos, modos de pensar, en una palabra, todo eso que se llama concepción del mundo de un grupo y que tan hondamente puede afectar su conducta económica, entran a llenar ese esqueleto de fórmulas más o menos vacías pero que en el fondo constituyen el pensar científico, y en el caso de la economía la verdadera ciencia económica. Los principios generales, los métodos, los conceptos de la economía como los de toda ciencia son universales y constituyen justamente un instrumental para poner orden en un material concreto que hemos de examinar desde el punto de vista de la escasez.

Es, pues, necesario insistir en que no hay una ciencia económica socialista, ni liberal ni conservadora, ni cristiana ni laica, porque todas estas son categorías aplicables a otras esferas de la realidad como la moral, las costumbres, las formas de vida políticas o las relaciones sociales, pero carecen totalmente de sentido para una clasificación de las ciencias. Y mientras nuestros medios docentes no estén servidos por especialistas saturados de algo que podríamos llamar una moral científica, mientras el rigor lógico y la visión clara del campo de problemas de la economía, de las necesidades del país y de las exigencias internas de la docencia no sean la base inconmovible de sus orientaciones, la enseñanza económica corre el riesgo de convertirse en apologética y de no salir jamás del plano de ensayos y confusiones en que, debemos reconocerlo, se ha movido hasta el presente. 